Nurse Practitioner Open Journal

Vol 1 DOI 10.28984/npoj.v1i1.34

\title{
Case presentation of Safe and Effective Use of Medical Cannabis in the Elderly
}

NPOJ 2021, Vol 1 , pp. 9-16

Luisa Barton , DNP, NP-PHC ${ }^{1}$

luisabarton@athabascau.ca https://orcid.org/0000-0002-5072-4168

Clara Nisan, MN, NP-PHC ${ }^{2}$

cnisan@universalcareinc.ca

Carey Burleigh, RSW ${ }^{3}$

mhltcsocialworker@universalcareinc.ca

Suzanne Fredericks ${ }^{3}$, RN, PhD

sfrederi@ryerson.ca https://orcid.org/0000-0002-7462-636X

${ }^{1}$ Faculty of Health Disciplines, Athabasca University, Alberta, Canada

${ }^{2}$ UniversalCare Canada Inc., Ontario, Canada

${ }^{3}$ Daphne Cockwell School of Nursing, Ryerson University, Ontario, Canada

\begin{abstract}
Introduction: The therapeutic benefits of medical cannabis have been demonstrated for a number of chronic conditions impacting the elderly population, such as pain management, and as alternatives to antipsychotic and opioid interventions, as well as end of life treatments. However, this therapeutic intervention has not become part of routine care for seniors living in long-term care facilities because of reduced public acceptance and stigma. The aim of this paper was to present case studies outlining the effective use of medical cannabis to treat elderly patients with a variety of medical conditions and symptoms such as: post traumatic stress disorder, pain, anxiety, delusions, as well as palliative care. Cannabis was also used to taper antipsychotic medications, and for managing those in palliative care.

Case Presentation: Three cases highlighting the use of medical cannabis are described, from the perspective of a nurse practitioner-led interdisciplinary team approach.

Management and Outcome: Using a variety of combinations of medical cannabis (cannabidiol and delta-9tetrahydrocannabinol) in oral formulations, the long-term care facility has achieved a dramatic reduction in the use of antipsychotic medications. Medical cannabis has shown alleviation of many symptoms such as: pain, dyspnea, agitation, fatigue, weakness, loss of appetite, nausea, vomiting, and twitching. Positive results were noted in several palliative care patients who received medical cannabis for pain and symptom management.

Conclusion: As an adjunct therapy for managing post traumatic stress disorder and other conditions, medical cannabis has been effective in reducing symptoms and for improving the patients' overall quality of life. Continued evaluation into the long effectiveness of medical cannabis provided to individuals over the age of 65 years is suggested. This nurse practitioner-led therapeutic intervention highlights the potential health benefits of medical cannabis and has clinical implications for practice and education.
\end{abstract}

Key words: medical cannabis, elderly, long-term care, pain, palliative care 


\section{Introduction}

Medical cannabis is becoming a promising therapy for the treatment of many health conditions impacting the elderly population. Some of these include chronic pain and responsive behaviours, which, in turn, may lead to deprescribing opioids and antipsychotic medications. Use of cannabis is also being used more often in palliative care. Nurse Practitioners (NPs) play a vital role in Canada's health care system. With their extensive and advanced knowledge and skill in various health care settings, NPs are well equipped to provide comprehensive care, including the provision of cannabis therapy for various medical indications. According to the College of Nurses of Ontario (2019), NPs who complete a written order or medical document authorizing the use of cannabis for medical purposes are expected to use evidence to inform their treatment decisions, as with all other therapeutic options. Specifically, NPs must also comply with the requirements under the Cannabis Regulations in Canada (College of Nurses of Ontario [CNO], 2019). Other jurisdictions in Canada also authorize NPs to complete a medical document for the use of medical cannabis as a therapeutic intervention. Given this, it is anticipated that experiential clinical knowledge and further research in the field of medical cannabis can better inform health outcomes of all Canadians.

In Canada, cannabis consumption among those greater than 65 years old has been accelerating at a much faster pace than it has among other age groups (Statistics Canada, 2019). Older adults and the elderly encounter a number of medical issues that are not solely alleviated by conventional pharmacological agents. Symptoms such as poor sleep, pain, anxiety, and delusions from diagnoses, such as dementia and PTSD, are too often not fully resolved (Ahmed et al., 2015) Although research continues to be limited, recent studies are showing promising results from the use of cannabis therapy. According to Abuhasira et al. (2018), the treatment of medical cannabis in elderly patients suggests that cannabis is effective in improving pain and quality of life. With regards to dementia, cannabinoids may be a beneficial therapy for responsive behavioural symptoms, such as agitation, and other neuropsychiatric symptoms (Ahmed et al., 2015; Katz, Katz, Shoenfeld \& Porat-Katz, 2017). While there is a significant lack of robust research, a recent study by Minerbi et al. (2019) describes that cannabinoids work on a chemical level to target pain, psychiatric issues, and sleep.

As NPs consider using cannabis therapy for the elderly population in long term care facilities, studies are emerging regarding its use. For example, Palace \& Reinfold (2019) examine ways in which cannabinoids are used to treat elderly patients using a case study approach. The study focuses on decreasing prescription medications for patients who rely on opioids for pain management and other health challenges. Similar to the Palace \& Reinfold (2019) case study approach, this paper will also use clinical cases to examine how medical cannabis has been effectively used in an Ontario Long-Term Care (LTC) Facility to treat elderly patients with a variety of medical conditions and symptoms.

\section{The Endocannabinoid System}

The endocannabinoid system (ECS) is a complex and not fully understood system that involves three core components: endocannabinoids, receptors, and enzymes. Endocannabinoids, 
also called endogenous cannabinoids, are molecules produced by the body. There are two key endocannabinoids: anandamide (AEA) and 2-arachidonoylglyerol (2-AG). With regards to receptors, $\mathrm{CB} 1$ and $\mathrm{CB} 2, \mathrm{CB} 1$ receptors are more common in the central and peripheral nervous systems and the gastrointestinal system, while CB2 receptors cluster in the immune system, including the spleen and lymph nodes (Hatcher et al., 2018; Lu \& Mackie, 2016). Via the CB1 and $\mathrm{CB} 2$ receptors, cannabinoids act on a number of pathways in the body, which allow them to affect systems as diverse as feeding/hunger, insulin sensitivity, stress responses, gut permeability, inflammation, and emotional states (Hatcher et al., 2018).

Enzymes are responsible for breaking down endocannabinoids once they've carried out their function. There are two main enzymes responsible for this: the fatty acid amide hydrolase, which breaks down AEA and monoacylglycerol acid lipase, which typically breaks down 2-AG. The two most notable compounds to medical cannabis are THC (delta-9-tetrahydrocannabinol) and CBD (cannabidiol). THC exerts its effect primarily through the activation of CB1, however, $\mathrm{CBD}$ does not bind to either $\mathrm{CB} 1$ or $\mathrm{CB} 2$ but rather, it inhibits or activates other receptors, enzymes, and molecules (Lu \& Mackie, 2016). Although there is still much to know about the ECS, understanding how CBD and THC affects these systems is paramount to using medical cannabis to benefit various health conditions.

\section{Medical Cannabis: Compounds, Formulations and Dosing}

There are several hundred natural compounds, including over 120 cannabinoids that have been isolated from cannabis species. Both THC (delta-9-tetrahydrocannabinol) and CBD (cannabidiol) (Hatcher et al., 2018) are most relevant in medical cannabis. THC is responsible for many of the psychoactive effects of cannabis whereas CBD does not have the euphoric effects. Cannabis is available in a variety of formulations including dried cannabis, oils, soft gels, and capsules. Notwithstanding the strength of these formulations, the modes of administration, whether by inhalation or ingestion, is also an important factor. Many licensed producers of cannabis classify their formulations as short and long acting; ingested cannabis has a duration effect of 6-10 hours with its onset of 1-3 hours whereas inhalation formulations have a shorter onset (5-10 minutes) and effect (2-4 hours) (Hatcher et al., 2018).

\section{Case Presentations}

The following three case presentations are based in a Long-Term Care (LTC) Facility in Ontario, Canada. The facility is a 170-bed, non-profit home with a strong supportive leadership team and it has become a front runner in innovative practices. Currently, the residents that are entering LTC facilities has shifted from elderly individuals who are somewhat independent and are not in the end stages of their disease trajectory to those who are very frail and have significant responsive behaviours, pain management requirements, and are at the end of their lives. In 2017, a senior level NP, along with an interdisciplinary team in their LTC unit began exploring the use of medical cannabis. The impetus for this was largely attributed to the physical and mental complexity of the population served in the LTC and the need for innovative approaches to pain and symptom management given that mainstream medicine may not always be the most effective or best way to manage residents' issues. Using the LTC's medical cannabis 'prescribing' protocols, based on evidence from a variety of peer-reviewed journals and medical 
expertise, guiding principles were followed such as; "start low, go slow"; the older and more medically complex the patient, the lower the THC and the higher the CBD ratio; titrate by increasing the dose by $1.25 \mathrm{mg}$ THC daily and/or $2.5 \mathrm{mg}$ CBD until the resident has sufficient symptom response and/or has reached the maximum authorized daily dose and/or the resident experiences adverse effects (Ahmed et al., 2015; Hatcher et al., 2018; Lim, See \& Lee, 2017; MacCallum \& Russo, 2018). The following three case studies illustrate the positive impact of medical cannabis use, as NP-led intervention, in the LTC.

\section{Case A}

A 91- year -old female resident suffered from several co-morbidities such as dementia, delusion disorder, urinary tract infections, arthritis, and intervertebral disc degeneration. The resident also had an unresolved delirium that followed a fall, resulting in a fracture. Due to her history of severe mental trauma as a Holocaust survivor, she was diagnosed with Post-Traumatic Stress Disorder (PTSD). She experienced horrific visual hallucinations that left her feeling terror. She had multiple falls and would not eat or drink which resulted in significant weight loss. Unfortunately, the resident frequently yelled out in fear due to these hallucinations.

The inter-professional team and her family tried all interventions available to them. Different medications were trialed and she was referred to the Behaviour Support Services mobile team multiple times. A panel of experts, through Behaviour Supports Ontario, reviewed her complex case and she was admitted to a specialized behavioural neurology unit in one of the leading hospitals in the geographical area for six weeks. All efforts were unsuccessful. She returned from the hospital to the LTC in a further declined condition. The inter-professional team and the resident's family decided she would only benefit from a palliative approach to care.

Using a holistic approach, the NP explored the use of medical cannabis as an alternative to addressing the resident's delirium, poor appetite, restlessness, agitation, and pain. The resident's family was willing to trial this intervention and the resident was started on CBD (cannabidiol) oil in December 2017. Given that the resident had episodes of delirium, THC was not recommended. Using the 'start low and go-slow approach' with elderly people, the daily amount prescribed was $0.5 \mathrm{gm} /$ day CBD dominant oil (CBD $20 \mathrm{mg} / \mathrm{ml}$; THC $<1 \mathrm{mg} / \mathrm{ml}$ ) $0.5 \mathrm{ml}$ ingested once daily then slowly increasing to twice daily for 3 months.

The effects of the cannabis treatment were noted quickly. Within weeks, the resident began to eat and eventually feed herself, subsequently leading to weight gain. Even more notably, her violent visual and auditory hallucinations ceased, and she began to communicate with her family and LTC staff. The resident no longer fell from her bed as she once did, and she began to sleep through the night. According to her pain scale, pain from her arthritis eased significantly from 8/10 (high pain level) to 4/10 (low to moderate pain level). To date, medical cannabis has been the only effective therapeutic option for this resident and in doing so, four important health outcomes were achieved: pain reduction, improved appetite, decreased hallucinations, and improved sleep. 


\section{Case B}

An 86-year-old female resident, who was suffering from ophthalmic shingles, had additional diagnoses of dementia, diabetes, congestive heart failure, delusional disorder, osteoporosis, and COPD. The shingles caused severe pain, resulting in an overall decline and poor intake of food and fluid. Common pain medications were trialed with little improvement. Medical cannabis was offered as an option to address pain, stimulate her appetite, and to decrease agitation. The resident's family agreed and medical cannabis was started using CBD (cannabidiol) oil in November 2018. The dose prescribed was 1gm/day of CBD oil (CBD $25 \mathrm{mg} / \mathrm{ml}$; THC $<0.5 \mathrm{mg} / \mathrm{ml}$ ) starting with $0.5 \mathrm{ml}$ ingested once daily to gradually $1 \mathrm{ml}$ twice daily. The resident's pain began to improve from $7 / 10$ to 3/10 in her pain scale. In addition, her appetite improved significantly, she became more interactive with others. To date, she continues to thrive using the same regimen of medical cannabis.

\section{Case C}

A 73-year-old female resident, who had a long history of dependence and misuse of opioids since the age of 18 , also had several co-morbidities including kidney failure with dependence on renal hemodialysis, diabetes, and paranoid personality disorder. She also suffered from chronic neuropathic pain. The resident was previously prescribed multiple opioids, including Hydromorphone and Fentanyl. She frequently requested dimenhydrinate and diphenhydramine to help her sleep for longer, uninterrupted periods of time. The resident's demeanor included drowsiness, refusal to eat, and inability to engage with others and activities.

As an initial harm reduction strategy (given her use of opioids), she was started on CBD oil and then had THC oil added to her regimen in November 2018. The resident was prescribed $1 \mathrm{gm} /$ day of blended THC and CBD (THC $10 \mathrm{mg} / \mathrm{ml}$; CBD $12 \mathrm{mg} / \mathrm{ml}$ ) $0.5 \mathrm{ml}$ oil ingested up to four times per day for a 3-month trial. During the three-month period of using medical cannabis, the resident was slowly tapered off of the Hydromorphone and Fentanyl (from a $75 \mathrm{mcg} / \mathrm{h}$ transdermal patch to $25 \mathrm{mcg} / \mathrm{h}$ transdermal patch) until she was completely weaned off all opioids, yet, with improved pain control.

The resident's mood also slowly improved as she became more alert and able to interact with others without agitation or conflict. The resident's health outcomes included opioid/harm reduction, improved pain management, and improved mood. Unfortunately, the resident passed away due to kidney failure, however, it was determined by the LTC staff that she was able to experience an overall improved quality of life at the end of her life.

\section{Management and Outcome}

Health Canada asserts that "medical cannabis refers to the use of cannabis and its constituents including cannabinoids, tetrahydrocannabinol (THC) and cannabidiol (CBD), to alleviate symptoms of specified diseases" (Health Canada, 2018, para 1). The optimal dose of medical cannabis should be symptom improvement and functioning while causing minimal euphoria or cognitive impairment. The potential for adverse effects in the use of cannabis in the elderly warrants the general approach of "start low, go slow, and stay low" when it is introduced 
in a resident's treatment plan. This basic principle was adopted in the medical cannabis protocol of the aforementioned case studies.

For the general population, the average dose of medical cannabis is $0.5-3$ grams per day (Hatcher et al., 2018). Depending on age and indication for medical cannabis, CBD can be either used alone or in combination with THC. Often, with the elderly population, THC combined with CBD has been noted as effective for sleep, hence, often used at bedtime (Hatcher et al., 2018; MacCallum \& Russo, 2018). For pain, CBD alone or in combination with a low amount of THC for an 'entourage effect' is often recommended. A slow upward dose titration promotes tolerance to the psychoactive effects of THC. Significant drug interactions with cannabis are rare and for the elderly, this is very important given concern over polypharmacy. Given the facility's smokefree policy, the only medical cannabis formulation used for this NP-led intervention was oral ingestion of both CBD and THC oils; this formulation is considered long-acting therapy.

To date, the LTC facility continues to utilize medical cannabis for palliative symptom management, chronic pain, neuropathic pain, poor appetite, sleep issues, responsive behaviours, hallucinations, delusions, and anxiety for its residents. The provision of cannabis has been tailored and slowly tapered for residents on antipsychotic medication while closely monitoring and evaluating residents' symptoms and behaviours. As a result, the LTC has achieved a dramatic reduction in the use of antipsychotic medication from the provincial average of $19.3 \%$ to $11.6 \%$.

In the NP's experience, palliative residents can have many troubling symptoms including: pain, dyspnea, terminal agitation, and/or terminal secretions. Additional symptoms can include fatigue, weakness, altered level of consciousness, loss of appetite, nausea, vomiting, jerking or twitching. Medical cannabis has shown alleviation of these symptoms. Also, medical cannabis continues to be used for several residents receiving palliative care. In conjunction with hydromorphone, midazolam and scopolamine, medical cannabis is being offered as an adjunct therapy. In these cases, residents have remained comfortable through the dying process.

\section{Discussion}

The authorization of medical cannabis in the LTC facility has been transformational to its residents. Since 2017, the number of residents receiving prescribed cannabis has grown to a total of 60 residents. Of the 170 residents in care, only 8 are now being given antipsychotic medication without appropriate diagnosis. The interprofessional team members were able to acquire greater knowledge about medical cannabis and apply it to their practice in the hope that it would make a difference in the overall quality of life of their patients. Monitoring and evaluation of medical cannabis as an intervention continues to be ongoing, however, to the interprofessional team and the residents (as well as their families), the use of medical cannabis has been regarded as a success story. There have been no reports from residents, family, or staff of any adverse effects of using medical cannabis. To date, it has been used to treat pain, poor appetite, agitation, restlessness, anxiety, depression, poor sleep, or in conjunction with medication for active palliative care. Furthermore, although most of the physicians were initially hesitant to consider medical cannabis as a therapeutic option, more physicians are now on board with its use given 
the positive health outcomes noted in the residents. It is hoped that this is a step in the right direction for de-stigmatizing medical cannabis use.

After three years of anecdotal data collection and monitored use of medical cannabis, the LTC has also concluded that the geriatric population in long-term care is a safe cohort to use medical cannabis. This is primarily due to the availability of $24 / 7$ supervision by the interprofessional team, having access to an on-site prescriber/authorizer and medical cannabis' safety profile. It is the interprofessional team's hope that more studies regarding the use of medical cannabis in the elderly population will be forthcoming.

\section{Conclusion}

This LTC is one of the first LTC homes in Ontario to adopt the use of medical cannabis and this nurse practitioner-led therapeutic intervention highlights the potential health benefits of medical cannabis and has clinical implications for practice. Although there is considerable experiential knowledge gained regarding the use of medical cannabis in residents of LTCs, more research studies are required to support its use as an adjunct therapy for a variety of medical conditions and symptoms. Continued evaluation into the long-term effectiveness of medical cannabis provided to individuals over the age of 65 years is suggested.

Health care professionals require more education in order to shift their practice to incorporate medical cannabis as a therapy for various medical conditions and symptom management. Although there is still much to know about the endocannabinoid system specifically, understanding how CBD and THC affects these systems is paramount to using medical cannabis to benefit individuals' health conditions. It is critical to note that information about the endocannabinoid system has been either lacking or completely omitted in the curricula of many health professionals' education. There are several reasons for this, including political barriers and the acceptability of medical cannabis in science (Evanoff et al., 2017). Nevertheless, it is imperative that all health care practitioners have a solid understanding of the endocannabinoid system, as well as some basic understanding of medical cannabis, irrespective of whether their practice will include offering medical cannabis as a therapeutic option.

Nurse practitioners, in particular, are in an optimal position to offer their patients holistic and comprehensive care guided by evidence-informed practice. Clinician bias coupled with limited education pertaining to medical cannabis pose as real barriers towards optimizing therapeutic interventions for patients who are struggling with chronic conditions. It is hoped that this case presentation paper will further open the discussion of medical cannabis in clinical practice as well as stimulate more research studies and policies in health care environments.

\section{References}

Abuhasira, R., Schleider, L.B., Mechoulam, R., \& Novack, V. (2018). Epidemiological characteristics, safety and efficacy of medical cannabis in the elderly. European Journal of Internal Medicine, 49, 44-50. doi: 10.1016/j.ejim.2018.01.019 
Nurse Practitioner Open Journal

Vol 1 DOI 10.28984/npoj.v1i1.34

Ahmed, A., van den Elsen, G., Colbers, A., Kramers, C., Burger, D., van der Marck, M., \& Olde Rikkert, M. (2015). Safety, pharmacodynamics, and pharmacokinetics of multiple oral doses of delta-9-tetrahydrocannabinol in older persons with dementia.

Psychopharmacology, 232, 2587-2595. doi: 10.1007/s00213-015-3889

College of Nurses of Ontario. (2019). Practice Standard. Nurse Practitioner. 1-16.

Evanoff, A. B., Quan, T., Dufault, C., Awad, M., \& Bierut, L. J. (2017). Physicians-intraining are not prepared to prescribe medical marijuana. Drugs and Alcohol Dependence, 180, 151-155. doi: 10.1016/j.drugalcdep.2017.08.010

Health Canada. (2018). Medical use of cannabis. Retrieved from: https://www.canada.ca/en/health-canada/topics/cannabis-for-medicalpurposes.html

Hatcher, L., MacCaullum, C., \& Schecter, D. (2018). Insights into cannabis-based medicines. Medical Cannabis 2018 Conference. Proceedings presented at 2018 Medical Cannabis Conference, Vienna, Austria.

Katz, I., Katz, D., Shoenfeld, Y., \& Porat-Katz, B.S. (2017). Clinical evidence for utilizing cannabinoids in the elderly. The Israel Medical Association Journal: IMAJ, 19(2), 7175 .

Lim, K., See, Y., \& Lee, J. (2017). A systematic review of the effectiveness of medical cannabis for psychiatric, movement and neurodegenerative disorders. Clinical

Psychopharmacology and Neuroscience, 15(4), 301-312. doi: 10.9758/cpn.2017.15.4.301

Lu, H., \& Mackie, K. (2016). An introduction to the endogenous cannabinoid system. Biological Psychiatry, 79(7), 516-525. doi: 10.1016/j.biopsych.2015.07.028

MacCallum, C.A., \& Russo, E.B. (2018). Practical considerations in medical cannabis administration and dosing. European Journal of Internal Medicine, 49, 12-19. doi: $10.1016 /$ j.ejim.2018.01.004

Minerbi, A., Häuser, W., \& Fitzcharles, M. (2019) Medical cannabis for older patients. Drugs \& Aging, 36(1), 39-51. doi: 10.1007/s40266-018-0616-5

Palace, Z.J., \& Reinfold, D.A. (2019). Medical cannabis in the skilled nursing facility: A novel approach to improving symptom management and quality of life. Journal of the American Medical Directors Association, 20(1), 94-98. doi: 10.1016/j.jamda.2018.11.013.

Statistics Canada. (2019). National Cannabis Survey, third quarter 2019. Retrieved from https://www150.statcan.gc.ca/n1/daily-quotidien/191030/dq191030a-eng.htm 